\title{
NLTE accretion disc models for the AM Canum Venaticorum systems
}

\author{
M. R. Nasser ${ }^{1}$, J.-E. Solheim ${ }^{1}$, and D. A. Semionoff $f^{2, \star}$ \\ 1 University of Troms $\varnothing$, Department of Physics, 9037 Troms $\varnothing$, Norway \\ 2 Institute of Theoretical Physics and Astronomy, A. Goštauto 12, Vilnus 2600, Lithuania
}

Received 30 January 2001 / Accepted 10 April 2001

\begin{abstract}
We present models of the optical spectra of two types of accretion disc atmospheres: a pure helium model and a $\mathrm{H}-\mathrm{He}$ model, in a low mass ratio, helium transferring, interacting binary white dwarf system. The computations are restricted to stationary discs in LTE and NLTE cases. We also investigate the influence on the spectra of some of the disc parameters such as the mass accretion rate, the angle of inclination, the $\mathrm{H} / \mathrm{He}$ mass ratio, and the inner and outer radius of the disc. Departures from LTE are also investigated in order to assess the necessity of computing more complicated and more time consuming models. We found that differences in predicted equivalent widths of helium line computed in LTE and NLTE models range between 10 and 40 percent. Finally we compare our disc models with 4 of the AM CVn systems observed with the Nordic Optical Telescope: AM CVn, HP Lib, V803 Cen and CR Boo to determine their parameters as mass transfer rates and inclinations of their discs.
\end{abstract}

Key words. stars: novae, cataclysmic variables - accretion discs - individual: AM CVn, HP Lib, V803 Cen, CR Boo

\section{Introduction}

AM CVn stars are a very particular type of cataclysmic variable, also called helium cataclysmic. At present, we know of eight such systems: AM CVn, CR Boo, HP Lib, V803 Cen, CP Eri, GP Com, RX J1914.4+245, and KL Dra (Nelemans et al. 2001a). It is currently believed that they are interacting binary white dwarf systems (Faulkner et al. 1972) with a degenerate $\mathrm{C}-\mathrm{O}$ primary in which the secondary low mass object is semi-degenerate with $M_{2} \leq 0.084 M_{\odot}$ and is slowly losing mass as it is layer by layer wrapped off in a process transferring an almost a pure helium gas to the white dwarf primary, most likely in the equatorial region in which a helium accretion disc is formed.

Send offprint requests to: M. R. Nasser, e-mail: M.R Nasser@phys.uit.no

* Based on observations made with the Nordic Optical Telescope, operated on the island of La Palma jointly by Denmark, Finland, Iceland, Norway, and Sweden, in the Spanish Observatorio del Roque de los Muchachos of the Instituto de Astrofisica de Canarias. The data presented here have been obtained using ALFOSC, owned by the Instituto de Astrofisica de Andalucia (IAA) and operated at the Nordic Optical Telescope under agreement between IAA and the NBIfA of the Astronomical Observatory of Copenhagen.
The AM CVn family members have in common a Herich composition (no hydrogen lines seen). They are analogous to the H-rich cataclysmic variables, showing wide absorption lines and ultra-short photometric variability. The observed photometric periods are between 17 and $45 \mathrm{~min}$. If the periods observed are the orbital periods or the superhump periods, then the only solution for such systems is that both objects are degenerate, or that we have a degenerate/semi-degenerate pair (Nelemans et al. 2001a).

Some of the group members have distinct high and low states depending on the mass transfer rate. Thus AM CVn and HP Lib are in a permanent high state and have broad line absorption spectra, like those of the UX UMa subclass of $\mathrm{CVs}$ with a high mass transfer rate $\dot{M}$ through their discs. GP Com has strong emission lines typical of low $\dot{M}$ systems, and the others change from predominantly absorption line spectra in their high states to weak emission line spectra in their low states, as in the VY Scl subclass of the H-rich CVs.

In this paper we focus on the optical part of the spectra where the accretion disc dominates, and present observed spectra of four of the AM CVn systems fitted with theoretical disc spectra. In the previous work (Semionovas \& Solheim 1999), the computed model grid was created varying the mass transfer rate $\dot{M}$ and the angle of inclination $i$ 
of a model disc atmosphere with mainly solar abundances of elements, except for a 10 times greater $\mathrm{He} / \mathrm{H}$ ratio. These models did not fit all the lines in the observed spectra. Complications appeared at $\lambda 4340 \AA$ where $\mathrm{H} \gamma$ could be mistaken for He II at $\lambda 4338 \AA$ and in the $\lambda 4388 \AA$ and $\lambda 4471 \AA$ He I regions.

The most important result, presented later in this paper, is that the AM CVn stars must have an almost pure helium disc with a maximum mass ratio $\approx 10^{-5}$ of hydrogen with respect to helium, in order to avoid the problems mentioned above. The determination of $\dot{M}$ is also an important parameter to determine the evolutionary stage of these objects. The main parameters assumed to make changes in the global appearance of the spectra are the mass accretion rate, the angle of inclination, the inner and outer radius of the disc and the abundance. Similar work has been done by La Dous (1989) for the solar composition discs and by El-Khoury \& Wickramasinghe (2000) for the LTE helium-hydrogen disc. However this paper studies helium-hydrogen discs in both LTE and NLTE cases.

The paper is arranged as follows: in Sect. 2 we present the model atmospheres and state the basic assumptions and the equations used to calculate the vertical structure of an accretion disc. In Sect. 3 we set up the model grid of our calculations. The parameters which influence the disc spectra are presented in Sect. 4 , while in Sect. 5 we discuss the departures from LTE. Observations and data reduction techniques are presented in Sect. 6. In Sect. 7 we present the theoretical models which give the best fit to the observed spectra of V803 Cen, CR Boo, HP Lib and AM CVn. For AM CVn we also discuss other parts of the system using black body approximations. In Sect. 8 we discuss our results and in Sect. 9 we present our final conclusions.

\section{The model atmospheres}

In this section we will discuss the basic assumptions used to calculate the vertical structure of an accretion disc, and the basic equations included in the TLUSDISK code which are used in our calculations.

\subsection{The program}

In this work we present the fitted observed spectra of the AM CVn systems with theoretical disc spectra computed by the dedicated disc atmosphere model code created by I. Hubeny at Goddard Space Flight Center, NASA. The package includes several programs; the most important are the program for calculating vertical structure of the accretion disc TLUSDISK and the program for calculating an emergent spectrum based on a stellar or disc atmosphere model: SYNSPEC.

The program TLUSDISK is based on the model stellar atmosphere code TLUSTY (Hubeny 1988, 1990a,b). It is designed to calculate the vertical structure of a steady state equilibrium accretion disc and its atmospheres under the assumptions stated in the next section using the CL/ALI (Complete Linearization/Accelerated Lambda Iteration) method (Hubeny \& Lanz 1995). The user is given much freedom in choosing parameters for the calculation. This includes the possibility to calculate LTE and NLTE models of different levels of sophistication of atmospheres consisting of elements from hydrogen to zinc. Heavier elements can also be included in the model if that is necessary.

The first run of TLUSDISK produces an LTE model. If further refinement is needed, the output from this run can be used as a starting model for a model with NLTE treatment of continua and lines. The final output from TLUSDISK can be directed to SYNSPEC (Hubeny et al. 1994), which evaluates atmospheric opacity and emissivity and produces a synthetic spectrum. These two programs are accompanied by a set of utility and shell programs, which simplify the process of preparing the initial input and processing the output to give the final disc synthetic spectrum. In addition to that, we developed an utility program RINGRUN to simplify the production of a complete disc model (Semionovas 1998).

All the theoretical models we present in this paper are NLTE models. We will discuss the necessity of the NLTE treatment in more detail in Sect. 5 .

\subsection{Basic assumptions}

The disc is assumed to be in a steady state, geometrically thin and in keplerian rotation. The vertical structure is solved for a set of axially symmetric concentric rings where a plane-parallel 1-D atmosphere calculation is performed. The atmosphere at each disc radius $R$ (specified in the disc mid plane) is in hydrostatic equilibrium, with depth-dependent gravity $(g)$ that arises from the vertical component of the central star's gravitational force on the disc material. Neglecting the self-gravity of the disc and assuming that $R$ is much larger than the distance from the central plane $(z)$ :

$\frac{\mathrm{d} P}{\mathrm{~d} z}=-g(z) \rho$

$g=\frac{G M_{1} z}{R^{3}}$

where $P$ is the pressure, $\rho$ is the mass density, $M_{1}$ is the central star mass and $G$ is the gravitational constant. The mass surface density is given by the standard disc model (Shakura \& Sunyaev 1973):

$$
\begin{aligned}
& \Sigma=\frac{\dot{M}}{3 \pi \bar{\omega}}\left[1-\left(\frac{R_{1}}{R}\right)^{1 / 2}\right], \\
& \bar{\omega}=\frac{\sqrt{G M_{1} R_{1}}}{R e}
\end{aligned}
$$

where $\dot{M}$ is the mass transfer rate, $R_{1}$ is the central star radius, and $\bar{\omega}$ is the depth-averaged kinematic viscosity, parameterized in terms of Reynolds number $R e$ of the flow (Lynden-Bell \& Pringle 1974). It is useful to calculate the 
vertical atmosphere structure using the mass-depth variable $m(z)$, expressing the mass of a column of material above a given distance from the central plane $z$. The viscosity through the disc atmosphere can be expressed by a power law

$\omega(m)=\bar{\omega}(\zeta+1)\left(\frac{2 m}{\Sigma}\right)^{\zeta}$.

The power law index $\zeta$ is usually set to a positive nonzero value. The disc radiates all the mechanical energy dissipated by the viscous shearing between the keplerian orbits. According to the standard formulae this quantity may be written as follows:

$D_{\text {mech }}(z)=\frac{9 G M_{1} \omega(z) \rho(z)}{4 R_{1}^{3}}$

where $\omega(z)$ is the viscosity. The energy balance equation then becomes:

$\int_{0}^{\infty}\left(\kappa_{\nu} J_{\nu}-\eta_{\nu}\right) \mathrm{d} \nu=D_{\operatorname{mech}}(z)$

where $\kappa_{\nu}$ and $\eta_{\nu}$ are the absorption and emission coefficients, and $J_{\nu}$ is the mean intensity of the radiation, at frequency $\nu$.

As is customary in the stellar atmosphere theory, one expresses the total energy dissipated (and therefore radiated away) from the unit disc face as $D(R)=$ $\int D_{\text {mech }}(z) \mathrm{d} z$, through the effective temperature, $T_{\text {eff }}$ as $\sigma T_{\mathrm{eff}}^{4}=D(r)$, where $\sigma$ is the Stefan-Boltzmann constant. Assuming further a stationary, keplerian disc, the effective temperature is given by:

$T_{\text {eff }}=\left(\frac{3 G M_{1} \dot{M}}{8 \pi \sigma R^{3}}\left[1-\left(\frac{R_{1}}{R}\right)^{1 / 2}\right]\right)^{1 / 4}$.

Here is a summary of the basic assumptions:

- The energy balance is considered as a balance between the net radiation loss (calculated without invoking either optically thin, or optically thick approximations) and the dissipated mechanical energy;

- The dissipated energy is proportional to viscosity, which is given through the Reynolds number;

- The effect of illumination of the disc by the central star may be taken into account using the formalism described in Hubeny (1990b);

- The disc is not considered as a semi-infinite atmosphere but rather as a finite slab of gas. The optical thickness of the disc is a parameter that directly follows from the model;

- The total radiative flux is not constant, but increases upwards (from the central plane of the disc to its surface), its value being determined through the energy balance equation;

- Analogously, the gravity acceleration is not constant and depends on the distance from the central plane;

- The vertical structure of the disc (temperature, density, radiation field etc.) follows from the appropriate model calculations.
The program solves the basic equations (radiative transfer, hydrostatic equilibrium, energy balance, statistical equilibrium, charge and particle conservation) by the use of the hybrid CL/ALI (Complete Linearization/Accelerated Lambda Iteration) method (Hubeny \& Lanz 1995).

The thermal structure of the atmosphere, which is determined by the mass accretion rate and the radial distance from the central star, plays a crucial role in the convergence of the models, which consists of a set of concentric rings with variable $T_{\text {eff }}$, between the limits shown in Table 1.

\section{Setting the model grid}

Our goal of this modeling work is to find the best physical values for most of the disc parameters such as the mass accretion rate $\dot{M}$, the inner and the outer radii of the disc $R_{\text {in }}$ and $R_{\text {out }}$, the angle of inclination $i$ and the abundance of elements. An additional free parameter is the distance to the systems.

The first step is to prepare the initial grid of the disc models by specifying the mass of the central star $M_{1}$ with radius $R_{1}$, the mass accretion rate $\dot{M}$, the angle of inclination $i$, the abundance and the size of the disc. We will also study the effects of departures from LTE in order to assess the necessity of computing more complicated and more time-consuming NLTE models.

In this approach, we concentrate most of our work on pure helium models in order to investigate the possibility that the AM CVn systems may have a pure helium accretion disc, and then we compare our final results with the $\mathrm{H}$-He model of $\mathrm{H} / \mathrm{He}$ mass ratio $\approx 10^{-5}$.

For this work we have computed models with mass transfer rates $\approx(2-9) \times 10^{-9} M_{\odot} / \mathrm{yr}$, which is the range found to match the observed spectra of the AM CVn systems. We have used a model atom for helium with 14 levels of He I and one level of He II in the pure helium model. We also studied the spectra of the pure helium model when He II is included with 14 levels and He III as a highest one-level ion, and we found a small difference in the spectra of the very innermost rings of the disc when compared to the spectra of the same rings expected from the pure helium model of He II as a one-level ion. We have also found that the differences decrease gradually with increasing radial distance from the central star, and become completely negligible in the final integrated disc spectra. This is because He II is important in the innermost rings which make a small contribution to the total spectrum because of their small geometrical contribution (that is, $10 \%$ of the radial extent translates to only $1 \%$ of the total area). Moreover, He II lines that originate in the inner rings are smeared by Keplerian velocity which is largest there. Hydrogen, if considered, is represented by a 12-level model atom. All the atomic parameters (the photoionization cross-section, oscillator strength, collisional rates) are taken as default values provided by TLUSDISK. 
Parameters used in the calculations have ranges found realistic based on other investigations. For the disc size we use $R_{\text {out }}=(0.4-0.5) a$ (where $a$ is the distance between the two stars) which is about $15 R_{1}$, as a reference radius for both AM CVn and HP Lib. This is the disc size found for AM CVn (Solheim et al. 1998). For unstable systems like V803 Cen and CR Boo, the disc size according to the thermal-tidal-instability model of Tsugawa \& Osaki (1997) can oscillate within the three possibilities, the circularization radius (or the Lubow-Shu radius $R_{\mathrm{LS}}=0.29 a$ ), the critical radius for the tidal instability $R_{\mathrm{cr}}=0.46 a$ and the terminal disc radius $R_{\mathrm{d}}=0.36 a$ at the end of a superoutburst. This also gives an average disc radius of $15 R_{1}$ which we use as a reference radius for the discs of the AM CVn systems. A hole of $1.4 R_{1}$ is assumed to be in the center of the discs of the AM CVn systems to make room for a boundary layer, since almost all the AM CVn systems are X-ray sources (Warner 1995a). The He II line in emission at $\lambda 4686 \AA$ in the AM CVn systems is expected to appear due to the effect of the boundary layer in such systems (Robinson et al. 1992).

The final parameter is the mass of the central star presented in Table 3 which is assumed to be a fixed value, calculated using the mass-radius relation for a semidegenerate secondary filling its Roch lobe, presented by Savonije et al. (1986), and the computed value of $q=$ $M_{2} / M_{1}$ from the superhump period observations, or from the predicted values of this ratio based on the Savonije et al. (1986) mass-radius relation. The final disc models can have different angles of inclination from $i=0^{\circ}$ to $90^{\circ}$.

We are then left with only two varying parameters, the mass accretion rate $\dot{M}$ and the angle of inclination $i$, which are important for the physics and geometry of the systems.

\section{The influence of the parameters on the disc spectra}

In this section we study the influence of some of the accretion disc parameters on both the continuua and the line profiles of the accretion disc spectra. The parameters which on our investigation are focused are the mass accretion rate $\dot{M}$, the angle of inclination $i$ and the inner and outer radius of the disc $R_{\text {in }}$ and $R_{\text {out }}$. Another important parameter is the abundance of elements. We also include in this section Table 1, which shows the values of the effective temperatures at the inner and outer radius of the disc which varies with the mass accretion rate $\dot{M}$ and the mass of the central star $M_{1}$ (Eq. (8)).

\subsection{Mass accretion rate}

Figure 1 shows spectra of an accretion disc with different mass accretion rates $\left(\dot{M}=9,6\right.$ and $\left.3 \times 10^{-9}\right) M_{\odot} /$ yr. The radial temperature is a function of $\dot{M}$ (Eq. (8)), and as a consequence the continuum, becomes rapidly steeper with increasing $\dot{M}$. On the other hand, the lines become less deep with increasing $\dot{M}$, as seen in Fig. 2;
Table 1. The effective temperature $T_{\text {eff }}$ at the inner and outer radius of the disc with the variation of $\dot{M}$, while the mass of the central star is fixed at $M_{1}=1.1 M_{\odot}$ and $M_{1}=1.2 M_{\odot}$.

\begin{tabular}{lllll}
\hline$M_{1}=$ & $1.1 M_{\odot}$ & $1.1 M_{\odot}$ & $1.2 M_{\odot}$ & $1.2 M_{\odot}$ \\
$\dot{M}\left(M_{\odot} / \mathrm{yr}\right)$ & $R_{\text {in }}$ & $R_{\text {out }}$ & $R_{\text {in }}$ & $R_{\text {out }}$ \\
\hline $2 \times 10^{-9}$ & 68834 & 17196 & 78862 & 19701 \\
$3 \times 10^{-9}$ & 76177 & 19030 & 87275 & 21803 \\
$4 \times 10^{-9}$ & 81857 & 20449 & 93783 & 23429 \\
$5 \times 10^{-9}$ & 86554 & 21623 & 99164 & 24773 \\
$6 \times 10^{-9}$ & 90590 & 22631 & 103788 & 25928 \\
$7 \times 10^{-9}$ & 94149 & 23520 & 107866 & 26947 \\
$8 \times 10^{-9}$ & 97345 & 24319 & 111528 & 27862 \\
$9 \times 10^{-9}$ & 100254 & 25045 & 114861 & 28694 \\
\hline \hline
\end{tabular}

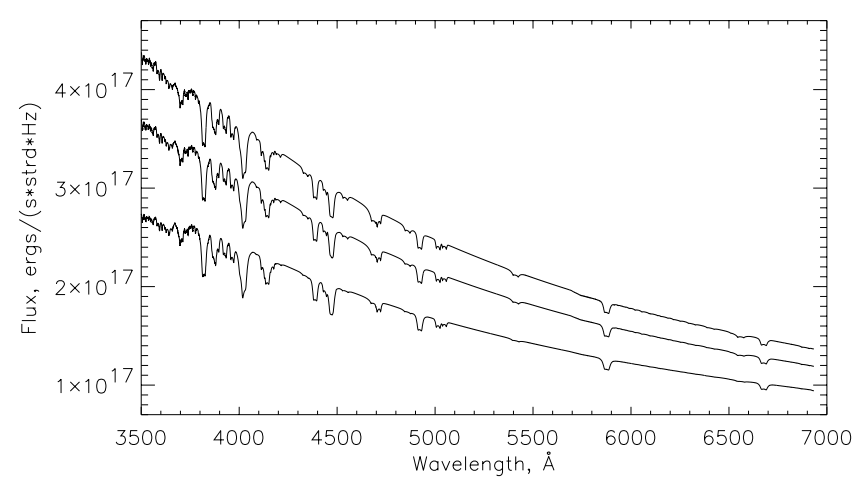

Fig. 1. The influence of the mass accretion rate on the slope of the NLTE pure helium accretion disc spectra. The parameters used for these models are $M_{1}=1.1 M_{\odot}, R_{\text {in }}=1.4 R_{1}, R_{\text {out }}=$ $15 R_{1}, i=20^{\circ}$ while $\dot{M}$ from the top is 9,6 and $3 \times 10^{-9} M_{\odot} / \mathrm{yr}$.

this is because for a higher mass accretion rate such as $\dot{M}=9 \times 10^{-9} M_{\odot} /$ yr the disc is hot everywhere $\left(T_{\text {eff }} \geq 25000 \mathrm{~K}\right.$ as shown in Table 1$)$, so the He I lines, which are strongest around $20000 \mathrm{~K}$, cannot contribute as much as in cooler discs where the He I are stronger for all annuli. The lines also become narrower with increasing $\dot{M}$ because the He I lines are formed in a given temperature range and thus originate mostly in the radially distant part of the disc where the Doppler broadening is smaller.

\subsection{Angle of inclination $i$}

Variations of the angle of inclination cause significant changes in the slope of the continuum, as shown in Fig. 3. The reason is because the total flux received from the disc increases with increasing $\cos (i)$. In addition, the lines are influenced in a more serious way as sharp, deep and narrow absorption lines are formed at smaller angles of inclinations. On the other hand, the line profiles become wider and filled with emission-like cores with even more narrow central absorption lines at higher inclinations, as seen in Fig. 4, where the angles considered are, from the top $75^{\circ}, 45^{\circ}$ and $15^{\circ}$. 

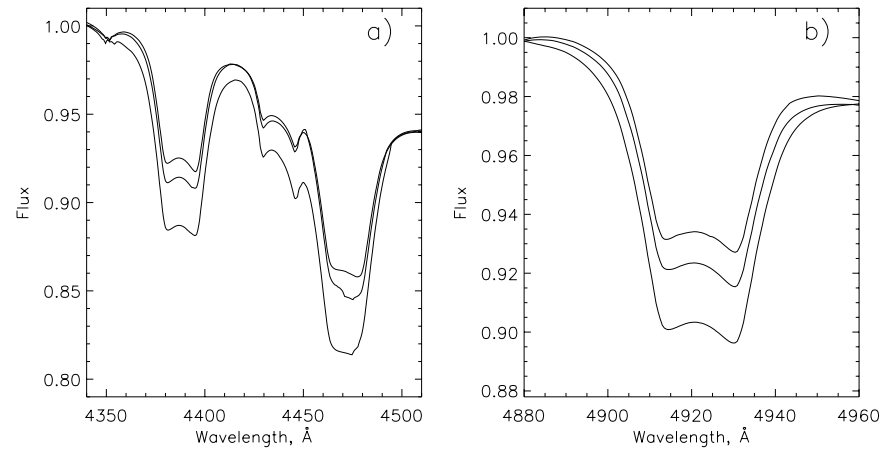

Fig. 2. The influence of the mass accretion rate on the line profile of the NLTE pure helium accretion disc spectra. The parameters used for these models are $M_{1}=1.1 M_{\odot}, R_{\text {in }}=$ $1.4 R_{1}, R_{\text {out }}=15 R_{1}, i=20^{\circ}$ while $\dot{M}$ from the top is 9,6 and $3 \times 10^{-9} M_{\odot} / y r$. The flux is normalised to 1.0 at the left hand upper corner.

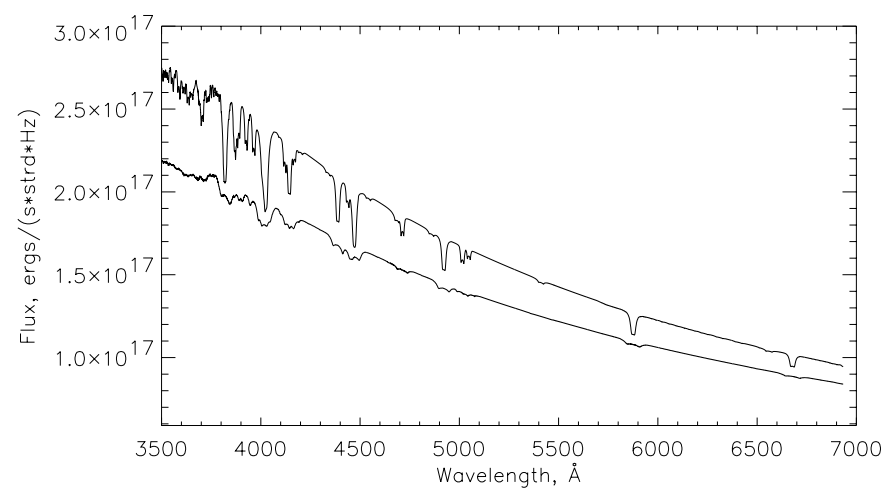

Fig. 3. The influence of the angle of inclination $i$ on the continuum of the NLTE pure helium accretion disc spectra. The parameters used for these models are $\dot{M}=3 \times 10^{-9} M_{\odot} / \mathrm{yr}$, $M_{1}=1.1 M_{\odot}, R_{\text {in }}=1.4 R_{1}, R_{\text {out }}=15 R_{1}$, while $i$ from the top $15^{\circ}$ and $75^{\circ}$.

\subsection{Inner radius of the disc}

We have studied the spectra of three different discs with $R_{\text {in }}$ of $1.4,4$ and $6 R_{1}$ while the rest of the system parameters remain the same: $\dot{M}=4 \times 10^{-9} M_{\odot} / \mathrm{yr}, M_{1}=$ $1.1 M_{\odot}, R_{\text {out }}=15 R_{1}$ and $i=20^{\circ}$. It is clearly seen in Fig. 5 that the slopes of the spectra become somewhat steeper with smaller $R_{\text {in }}$ of the disc, because of the added flux from the inner hot rings. On the other hand we found that the lines become deeper and with slightly narrower wings with larger $R_{\text {in }}$, as seen in Fig. 6. This is due to the removal of the inner hot rings with higher Keplerian velocities.

\subsection{Outer radius of the disc}

We have studied the influence of outer radius variations on the disc spectra, where we varied $R_{\text {out }}$ between 8,11 and $15 R_{1}$ while the inner radius at $1.4 R_{1}$ remains unchanged as are the rest of the system parameters. Figure 7 shows that the slopes of the spectra become steeper with smaller $R_{\text {out }}$ due to the higher flux received from the hot smaller
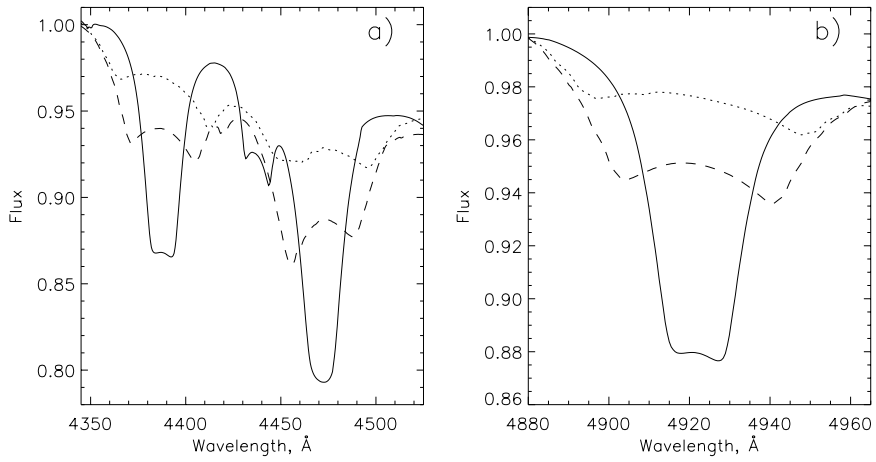

Fig. 4. The influence of the angle of inclination $i$ on the line profile of the NLTE pure helium accretion disc spectra. The parameters used for these models are $\dot{M}=3 \times 10^{-9} M_{\odot} / \mathrm{yr}$, $M_{1}=1.1 M_{\odot}, R_{\text {in }}=1.4 R_{1}, R_{\text {out }}=15 R_{1}$, while the angles represented by the (dotted line), (dashed line) and (solid line) are $75^{\circ}, 45^{\circ}$ and $15^{\circ}$ respectively The flux is normalised to 1.0 at the left hand upper corner.

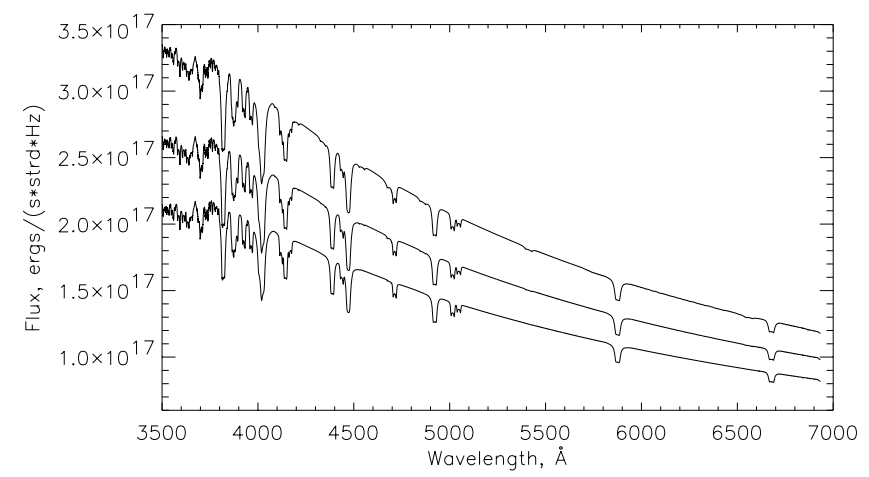

Fig. 5. The influence of the inner radius on the continuum of the NLTE pure helium accretion disc spectra. The parameters used for these models are $\dot{M}=4 \times 10^{-9} M_{\odot} / \mathrm{yr}$, $M_{1}=1.1 M_{\odot}, R_{\text {out }}=15 R_{1}$, and $i=20^{\circ}$ while the inner radius is from the top $1.4,4$ and $6 R_{1}$.

discs compared a cooler discs with a larger surface area. As in the $R_{\text {in }}$ variations case, the lines become slightly deeper and with a narrower core with bigger $R_{\text {out }}$, as in Fig. 8. This because of adding the outer cold rings with larger surface areas and lower effective temperatures and lines less affected by keplerian broadening.

\subsection{Abundance}

The abundance is one of the main parameters which influences the disc spectra. Since the AM CVn star's spectra can be explained with a pure helium model or may be a pure helium model with small traces of hydrogen, we investigated the influence of an extra amount of hydrogen on the line profiles. Although the observed spectra of AM CVn stars can be explained by pure-helium models, we investigated an influence of a small amount of hydrogen aiming to determine the upper limit of the hydrogen abundance. We found that even a small abundance of $\mathrm{H}$ can produce easily detectable features in the predicted spectrum. We demonstrate this in Fig. 9, which 

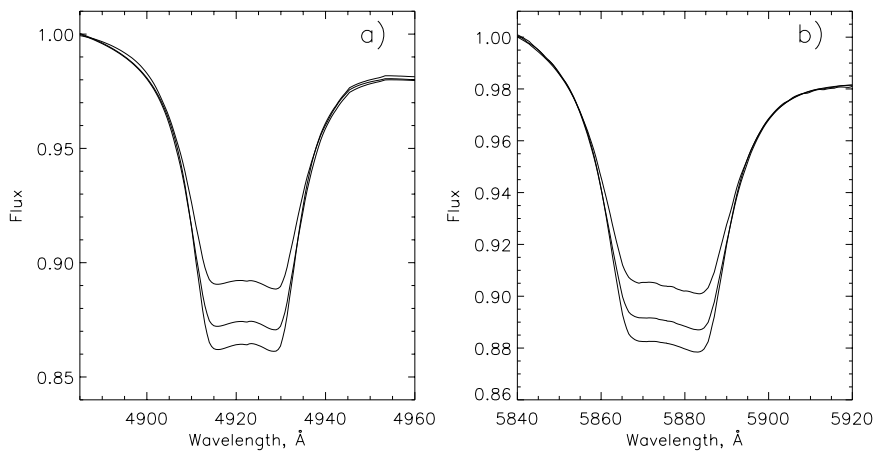

Fig. 6. The influence of the inner radius variation on the line profile of the NLTE pure helium accretion disc spectra. The parameters used for these models are $\dot{M}=4 \times 10^{-9} M_{\odot} / \mathrm{yr}$, $M_{1}=1.1 M_{\odot}, R_{\text {out }}=15 R_{1}$, and $i=20^{\circ}$ while the inner radius is from the top $1.4,4$, and $6 R_{1}$. The flux is normalised to 1.0 at the left upper corner.

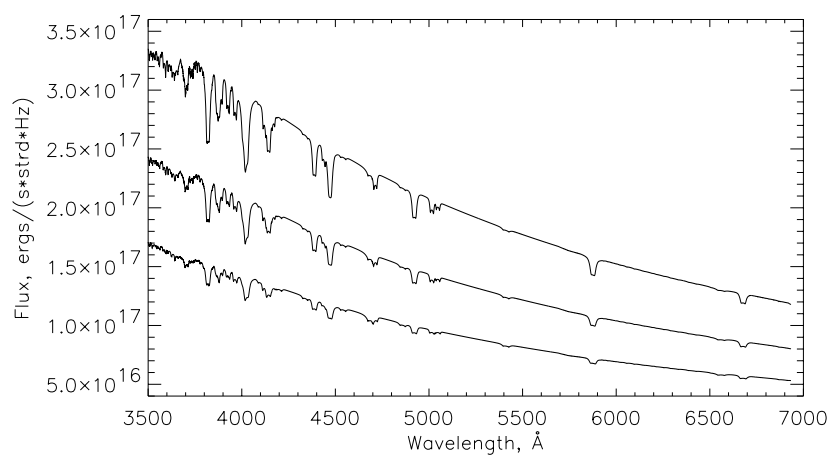

Fig. 7. The influence of the outer radius on the continuum of the NLTE pure helium accretion disc spectra. The parameters used for these models are $\dot{M}=4 \times 10^{-9} M_{\odot} / \mathrm{yr}$, $M_{1}=1.1 M_{\odot}, R_{\text {in }}=1.4 R_{1}$, and $i=20^{\circ}$ while the outer radius is from the top 15,11 and $8 R_{1}$.
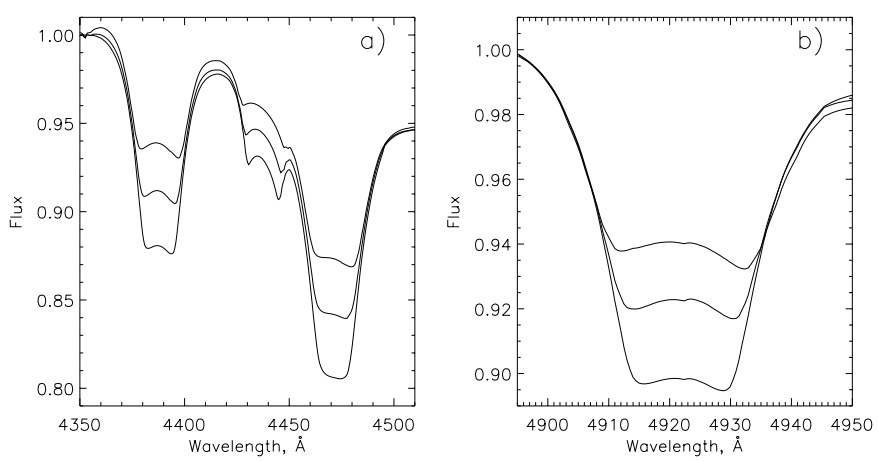

Fig. 8. The influence of the outer radius variation on the line profile of the NLTE pure helium accretion disc spectra. The parameters used for these models are $\dot{M}=4 \times 10^{-9} M_{\odot} / \mathrm{yr}$, $M_{1}=1.1 M_{\odot}, R_{\text {in }}=1.4 R_{1}$, and $i=20^{\circ}$ while the outer radius from the top is 8,11 and $15 R_{1}$. The flux is normalised to 1.0 at the left upper corner.

shows spectra for four values of hydrogen abundance, $\mathrm{H} / \mathrm{He}=10,10^{-1}, 10^{-3}$ and $10^{-5}$ (by mass). The computed spectra were convolved with a Gaussian profile with $F W H M=20 \AA$. Figure 9 thus shows the large sensitivity of the predicted spectrum to the hydrogen abundance,
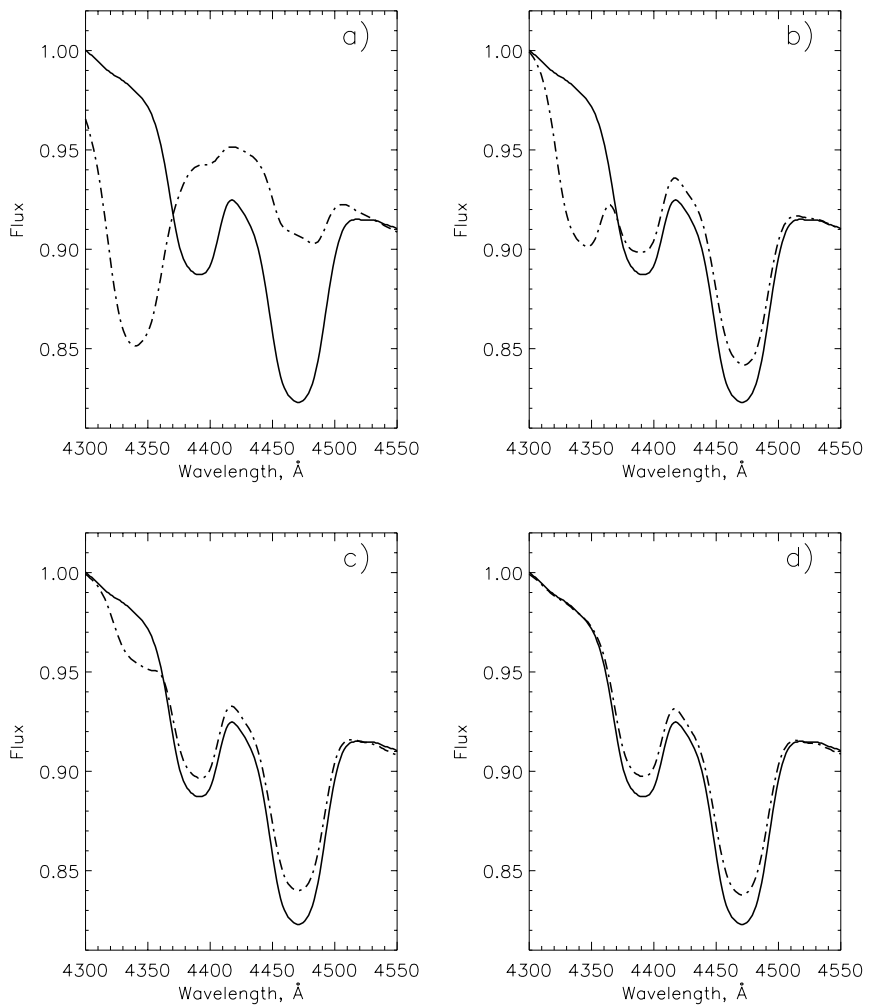

Fig. 9. The influence of an extra amount of hydrogen on the line profiles of the NLTE hydrogen-helium accretion disc spectra (dashed dotted line), when compared with the spectra of the pure helium model (solid line). The parameters used for these models are $\dot{M}=4 \times 10^{-9} M_{\odot} / \mathrm{yr}, M_{1}=1.1 M_{\odot}, R_{\text {in }}=$ $1.4 R_{1}, R_{\text {out }}=15 R_{1}$, and $i=35^{\circ}$ while the $\mathrm{H} / \mathrm{He}$ mass ratio varies from panel a) to $\mathbf{d}$ ) as $10,10^{-1}, 10^{-3}$ and $10^{-5}$, normalised to 1.0 at the left upper corner. The lines have been broadened with $F W H M=20 \AA$.

which makes it possible to estimate the upper limits of the $\mathrm{H} / \mathrm{He}$ mass ratios in the accretion disc from the observed spectra of the AM CVn systems, based on $20 \AA$ resolution spectra presented later in this paper.

\section{Departures from LTE}

For more than two decades, computing NLTE model stellar atmospheres, has demonstrated that NLTE effects play an important, even crucial role in atmospheres of virtually all types of hot stars (effective temperature around $10000 \mathrm{~K}$ and hotter); for a summary see Mihalas (1978); Kudritzki \& Hummer (1990) and Crivellari et al. (1991).

We have studied the effect of the LTE and NLTE treatment on the continuua and the line profiles of the pure helium disc spectra, and we found that the overall shape of the LTE and NLTE continua are very similar, as seen in Fig. 10. The He I lines, which are always in absorption in our models, are weaker in the NLTE compared to the LTE case, as can be seen in Fig. 11.

We found that the equivalent widths of He I lines in the NLTE case increase with decreasing mass accretion rate i.e. with decreasing temperature, and become comparable to the equivalent widths of the LTE lines at low 


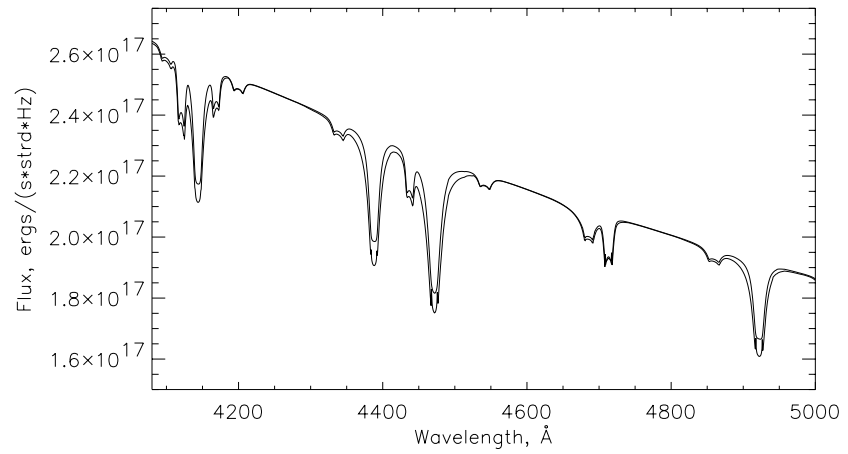

Fig. 10. The influence of the LTE and NLTE consideration on the continuum of the pure helium accretion disc spectra. Upper and lower lines represent the NLTE and LTE models respectively. The parameters used for these models are $\dot{M}=$ $4 \times 10^{-9} M_{\odot} / \mathrm{yr}, M_{1}=1.1 M_{\odot}, R_{\text {in }}=1.4 R_{1}, R_{\text {out }}=15 R_{1}$, and $i=10^{\circ}$.

temperatures. We expect that the He I lines may become even deeper in the NLTE than in the LTE case at very low temperatures, as obtained for the O stars by Auer \& Mihalas (1975). This was impossible to prove in our case because an average temperature lower than presented in Table 1, the test models did not converge because they were convectively unstable (due to low $T_{\text {eff }}$ ) and the current version of TLUSDISK does not treat convection properly. In order to get an idea of the difference in lines for models computed with LTE and NLTE, we have for a model grid calculated the difference in equivalent width of the He I line at $4388 \AA$. Denoting the line equivalent width in the LTE and NLTE case as $W_{\text {LTE }}$ and $W_{\text {NLTE }}$, respectively, we can define the relative difference between the two quantities as:

$\delta_{\mathrm{EW}}=\left|\frac{W_{\mathrm{LTE}}-W_{\mathrm{NLTE}}}{W_{\mathrm{NLTE}}}\right|$

Based on the value of $\delta_{\mathrm{EW}}$ we may explore differences between LTE and NLTE in any parameter space. We show this in $\dot{M}$ vs. $R$ space in Fig. 12 and in $\log (g)$ vs. $\log \left(T_{\text {eff }}\right)$ in Fig. 13. We have selected the pure helium models for these calculations.

In Figs. 12 and 13, contour lines correspond to the appropriate values of $\delta_{\mathrm{EW}}$ of the converged models, while shaded areas show the parameter space where the test models did not converge, most likely because they were convectively unstable due to low $T_{\text {eff }}$.

From Figs. 12 and 13 we conclude that the difference in equivalent width for the line selected can vary from less than 10 percent to more than 40 percent between LTE and NLTE models. We also conclude that disc models with pure helium composition must be treated as NLTE, in order to avoid discrepancies from 0.1 up to 0.4 in line equivalent width which may arise from LTE-only considerations.
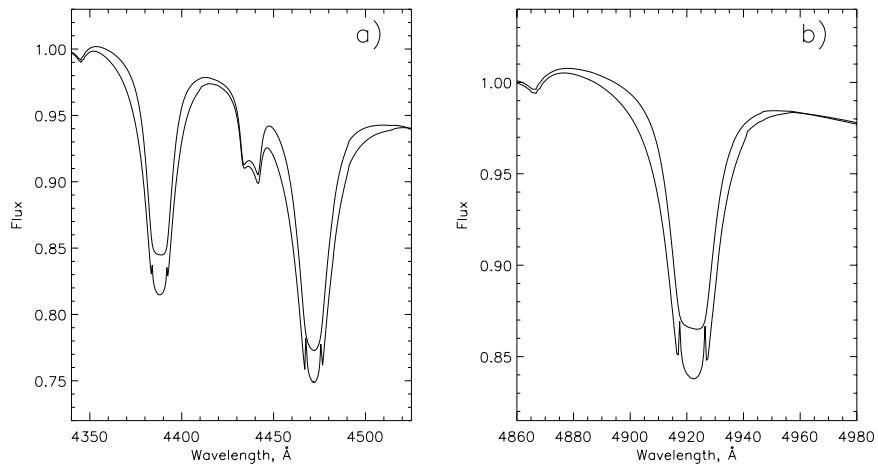

Fig. 11. The influence of the LTE and NLTE consideration on the line profile of the pure helium accretion disc spectra. Upper and lower lines represent the NLTE and LTE models respectively. The parameters used for these models are $\dot{M}=$ $4 \times 10^{-9} M_{\odot} / \mathrm{yr}, M_{1}=1.1 M_{\odot}, R_{\text {in }}=1.4 R_{1}, R_{\text {out }}=15 R_{1}$, and $i=10^{\circ}$, normalised to 1.0 at the left upper corner.

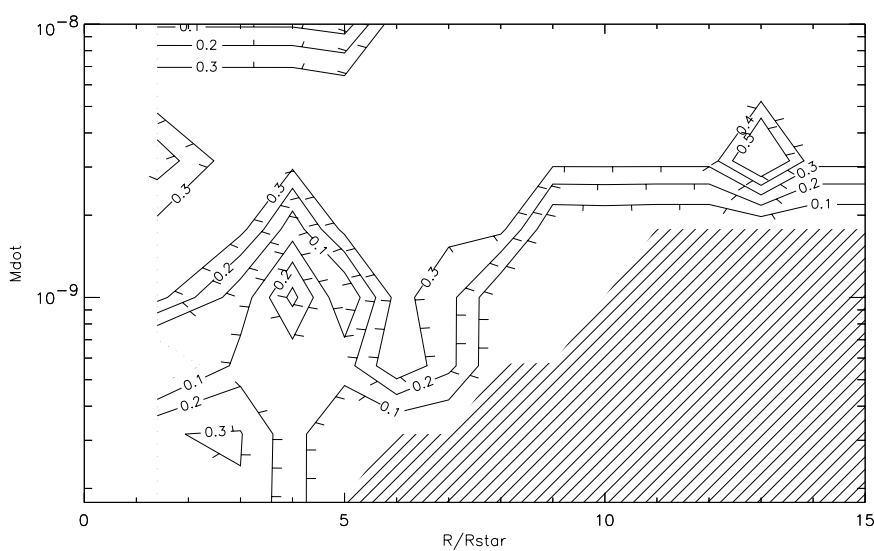

Fig. 12. Relative difference between the line equivalent width of the He I line at $\lambda 4388 \AA$ for LTE and NLTE calculations as a function of $\dot{M}$ and R.

\section{Observations and data reduction}

\subsection{Observations}

To investigate how well we can model helium discs for AM CVn objects, we observed the systems with the Nordic

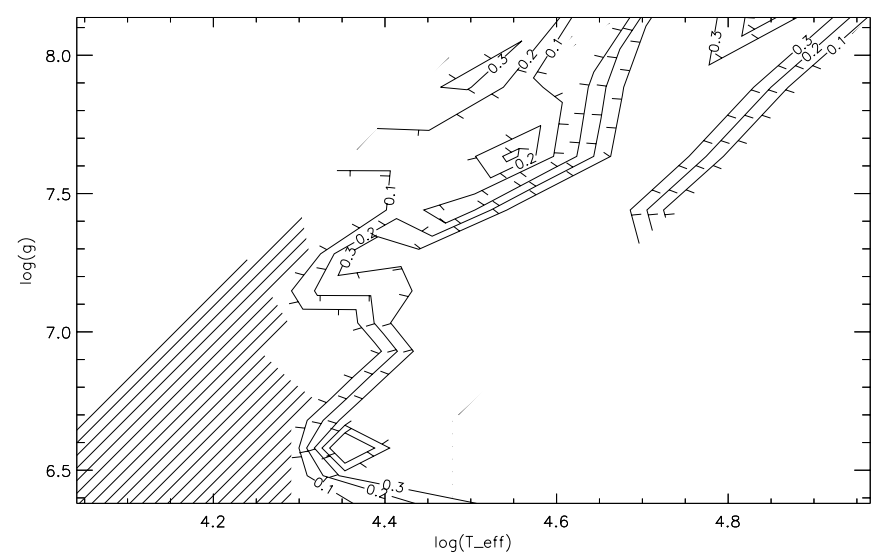

Fig. 13. Relative difference between the line equivalent width of the He I line at $\lambda 4388 \AA$ for LTE and NLTE calculations as a function of $\log (g)$ and $\log \left(T_{\text {eff }}\right)$. 
Table 2. List of spectra, obtained with the Nordic Optical Telescope in March 1997.

\begin{tabular}{|l|r|l|l|l|}
\hline File & Object & grism & slit & Ex.T. \\
\hline Name & Name & $\#$ & arcsec & Sec. \\
\hline gc010007 & GD 71 & 6 & 2.5 & 500 \\
gc010011 & PG 0943+441 & 4 & 2.5 & 200 \\
gc010015 & PG 1022+050 & 4 & 2.5 & 300 \\
gc010041 & AM CVn & 4 & 2.5 & 400 \\
gc010043 & AM CVn & 6 & 2.5 & 600 \\
gc010051 & GP Com & 4 & 2.5 & 600 \\
gc010052 & GP Com & 6 & 2.5 & 1200 \\
gc010056 & V803 Cen & 4 & 2.5 & 800 \\
gc010067 & CR Boo & 4 & 2.5 & 600 \\
gc010068 & CR Boo & 6 & 2.5 & 1200 \\
gc010076 & HP Lib & 4 & 2.5 & 400 \\
gc010080 & PG 1606+422 & 4 & 2.5 & 200 \\
gc010081 & PG 1606+422 & 6 & 2.5 & 400 \\
gc020051 & GD 71 & 6 & 0.7 & 1200 \\
gc020065 & PG 0943+441 & 6 & 0.7 & 1200 \\
gc020068 & AM CVn & 6 & 0.7 & 2400 \\
gc030014 & V803 Cen & 6 & 2.5 & 1600 \\
gc030015 & V803 Cen & 4 & 2.5 & 800 \\
gc030024 & HP Lib & 6 & 2.5 & 800 \\
gc030025 & HP Lib & 4 & 2.5 & 800 \\
gc030028 & PG 1606+422 & 6 & 2.5 & 800 \\
gc030029 & PG 1606+422 & 6 & 0.7 & 1200 \\
gc030084 & PG 0943+441 & 4 & 2.5 & 200 \\
gc030084 & PG 0943+441 & 4 & 2.5 & 200 \\
gc030085 & PG 0943+441 & 6 & 2.5 & 800 \\
gc030091 & AM CVn & 6 & 2.5 & 1200 \\
gc030092 & AM CVn & 4 & 2.5 & 800 \\
gc030094 & PG 1314+293 & 6 & 2.5 & 400 \\
gc030095 & PG 1314+293 & 6 & 0.7 & 1800 \\
gc030099 & GP Com & 4 & 2.5 & 800 \\
gc030100 & GP Com & 6 & 2.5 & 1800 \\
gc030102 & CR Boo & 4 & 2.5 & 1200 \\
gc030108 & V803 Cen & 4 & 2.5 & 800 \\
gc040007 & V803 Cen & 6 & 2.5 & 1600 \\
gc040018 & HP Lib & 6 & 2.5 & 800 \\
gc040020 & CR Boo & 6 & 2.5 & 2400 \\
gc040023 & HP Lib & 4 & 2.5 & 1400 \\
\hline
\end{tabular}

Optical Telescope (NOT), using the ALFOSC spectrograph for three nights from March 1st to 4th, 1997. The spectra should cover the longest possible continuous wavelength range to provide data for continuum fitting, and on the other hand have spectral resolution high enough for analysis of line profiles.

The reduced data presented in this paper were taken with a slit of 2.5 arcsec, which gave $20 \AA$ resolution. Spectrophotometric standard stars were chosen from the PG catalogue and the Hubble Space Telescope white dwarf calibration star list. The obtained spectra are listed in Table 2 .

\subsection{Data reduction}

The initial image processing and wavelength calibration were done using IRAF routines. The images were
Table 3. Observed parameters for some of the AM CVn systems.

\begin{tabular}{lllll}
\hline $\begin{array}{l}\text { Object } \\
\text { Name }\end{array}$ & $\begin{array}{l}P_{\text {orb }} \\
(s)\end{array}$ & $\begin{array}{l}\text { Mass Ratio } \\
M_{2} / M_{1}\end{array}$ & $\begin{array}{l}M_{1} \\
M_{\odot}\end{array}$ & $\begin{array}{l}\text { DiscSize } \\
R_{1}\end{array}$ \\
\hline AM CVn & 1028.27 & 0.084 & 1.1 & 15 \\
HP Lib & 1119 & $\geq 0.075$ & $>1.13$ & $(15-18)$ \\
CR Boo & 1471 & 0.057 & 1.035 & $(10-17)$ \\
V803 Cen & 1611 & $\leq 0.043$ & $<1.2$ & $(15-27)$ \\
\hline \hline
\end{tabular}

un-biased, zero corrected and then flat fielded. Frames were then illumination corrected using twilight sky flats and finally wavelength and flux calibrated. Simultaneous photometry showed that the unstable objects V803 Cen and CR Boo were in the state of rising brightness when observed. The CR Boo spectra, taken two days apart, still show almost constant colour index while many additional lines appeared in the brighter spectrum. In contrast, V803 Cen spectra, taken on March 2nd and 3rd, exhibit almost identical spectral line depths and profiles, while the increase in the $U$ and $B$ flux is much larger than in the $V$ and $R$.

The remaining three observed objects showed almost identical spectra from night to night, varying within the limits of observational errors. The spectra of AM CVn, V803 Cen, HP Lib and CR Boo show strong absorption lines at $\lambda 4338,4471,4922,5016$ and $5048 \AA$ while the remaining lines are either weak or blended, like $\lambda 4713$ and $\lambda 4438 \AA$. The continuum changes from one object to the other; AM CVn, CR Boo and V803 Cen show some fluctuations in the continuum at 5100-5400 $\AA$ which we are not able to identify as due to spectral lines or noise due to the sensitivity variations of the chip. These fluctuations did not appear in the spectrum of HP Lib. An absorption line at $\sim \lambda 4800 \AA$ appears in the spectra of all the objects presented in this paper; we suspect that this might be due to oxygen or nitrogen. In the following we compare our models with a subset of well flux-calibrated spectra.

\section{Observations compared with NLTE pure helium models}

In this section we fit only the pure helium accretion disc models with the observed spectra of the AM CVn systems and we find that the pure helium model gives a good fit to the observed spectra. In the next subsections we will discuss the fit to each system separately and discuss for one system (V803 Cen) the reasons for obtaining these good fits. We will also discuss possible errors in our results. Table 3 includes the observed and predicted values for some of the parameters of the AM CVn systems (Warner 1995b; Tsugawa \& Osaki 1997; Nelemans et al. 2001). 


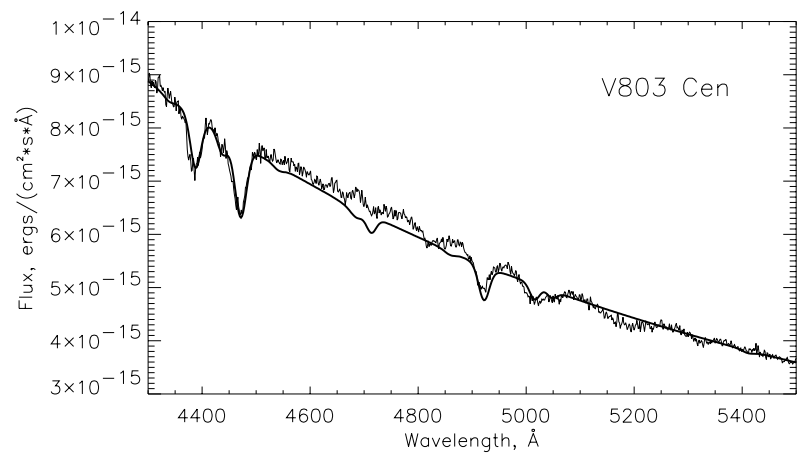

Fig. 14. The best NLTE model (solid line) which fits the observed spectrum "gc030014" (thick line) of V803 Cen with parameters $M_{1}=1.2 M_{\odot}, \dot{M}=3 \times 10^{-9} M_{\odot} / \mathrm{yr}, R_{\text {in }}=$ $1.4 R_{1}, R_{\text {out }}=17 R_{1}, i=5^{\circ}$.

\subsection{V803 Cen}

V803 Cen (=AE 1) was discovered by Elvius (1975) to be an unusually blue object in which the spectrum shows only broad, shallow absorption lines of He I with a complete absence of $\mathrm{H}$ lines. The He I features were found to vary strongly, fading to invisibility at times. It was classified as a rapid variable with a dominating period of $1611 \mathrm{~s}$ with a complex harmonic structure and a member of the AM CVn family by O'Donoghue et al. (1987).

Figure 14 shows the combination of parameters of the NLTE model which gives the best fit by the eye to the observed spectrum "gc030014" of V803 Cen. The values of the parameters are $M_{1}=1.2 M_{\odot}, \dot{M}=3 \times$ $10^{-9} M_{\odot} / \mathrm{yr}, R_{\text {in }}=1.4 R_{1}, R_{\text {out }}=17 R_{1}$ and $i=5^{\circ}$. The fit obtained with these values indicates that the distance of V803 Cen from the Earth is $\approx 405$ pc if we assume the absence of interstellar absorption.

This is not the only set of parameters which can give a fit similar to the one in Fig. 14; other combinations of parameters can also give a fit which is close to this one. We found that by varying one parameter, such as the mass accretion rate $\dot{M}$, within the range of $(3-5) \times 10^{-9} M_{\odot} / \mathrm{yr}$ as seen in Fig. 15, might give a similar but not a better fit than the one presented in Fig. 14. The same was noticed when we vary the angle of inclination and the size of the disc, as seen in both Figs. 16 and 17, respectively. Changing one of these parameters only and leaving the rest fixed will not give a better fit than the one presented in Fig. 14, but changing two or even three parameters at the same time might give a comparable fit to the one in Fig. 14. We found that we can get a similar fit to the one obtained in Fig. 14 with parameters within the following ranges: $\dot{M}=(4 \pm 1) \times 10^{-9} M_{\odot} / \mathrm{yr}, R_{\text {out }}=(17 \pm 3) R_{1}$ and $i=5^{\circ} \pm 5^{\circ}$, with the mass of the central star being fixed at $1.2 M_{\odot}$.

From such combinations of parameters for this system, we conclude that the size of the accretion disc of V803 Cen was (15-20) $R_{1}$ at the time of observations, which means the disc was within the range of the circularization and the tidal radius. We also found that the fit which we obtained for the disc size is closer to the circularization radius than

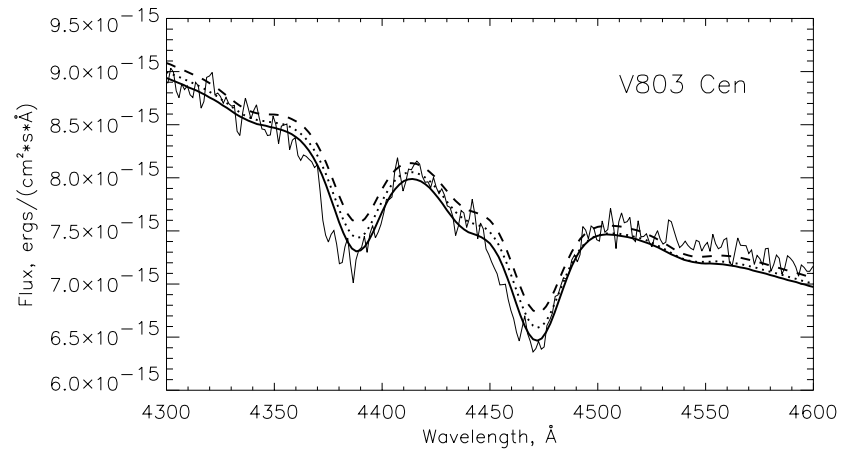

Fig. 15. The possible variations of the mass accretion rate, which can also give a satisfactory fit to "gc030014" compared to the one presented in Fig. 14, with the rest of the system parameters being fixed. Dashed, dotted and solid lines represent the mass accretion rate of 5,4 and $3 \times 10^{-9} M_{\odot} /$ yr respectively, when they are normalised at $\lambda 5500 \AA$.

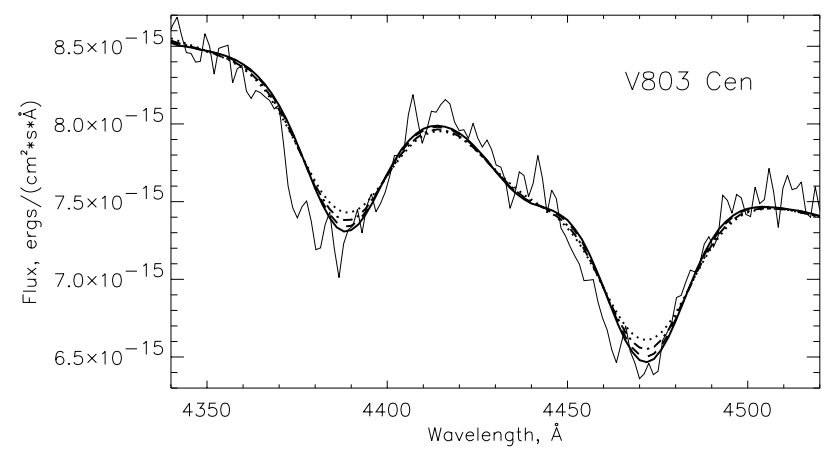

Fig. 16. The possible variations of the angle of inclination, which can also give a satisfactory fit to "gc030014" compared to the one presented in Fig. 14, with the rest of the system parameters being fixed. Dotted, dashed dotted, dashed and solid lines represent the angle of inclination of $15^{\circ}, 11^{\circ}, 8^{\circ}$ and $5^{\circ}$ respectively, when they are normalised at $\lambda 5500 \AA$.

the tidal radius, which confirms the finding during observation that the system was stable or may be progressing towards a rising phase. More well flux-calibrated spectra

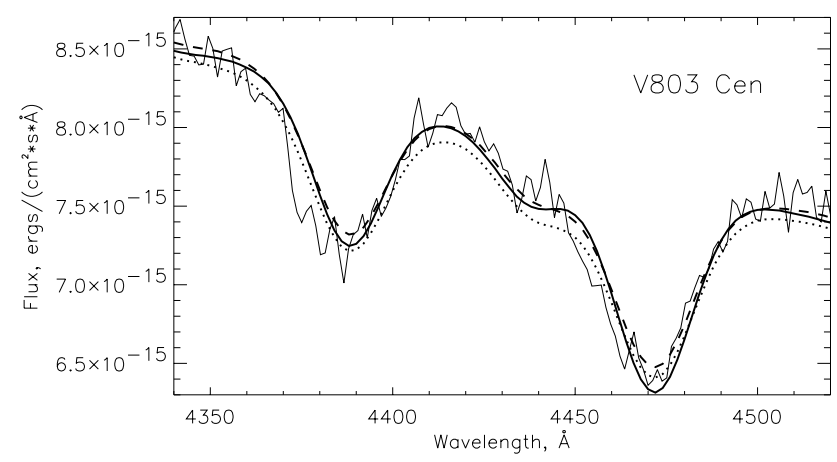

Fig. 17. The possible variations of the disc outer radius, which can also give a satisfactory fit to "gc030014" compared to the one presented in Fig. 14, with the rest of the system parameters being fixed. Dashed, solid and dotted lines represent the disc outer radius of 15,17 and $19 R_{1}$ respectively, when they are normalised at $\lambda 5500 \AA$. 


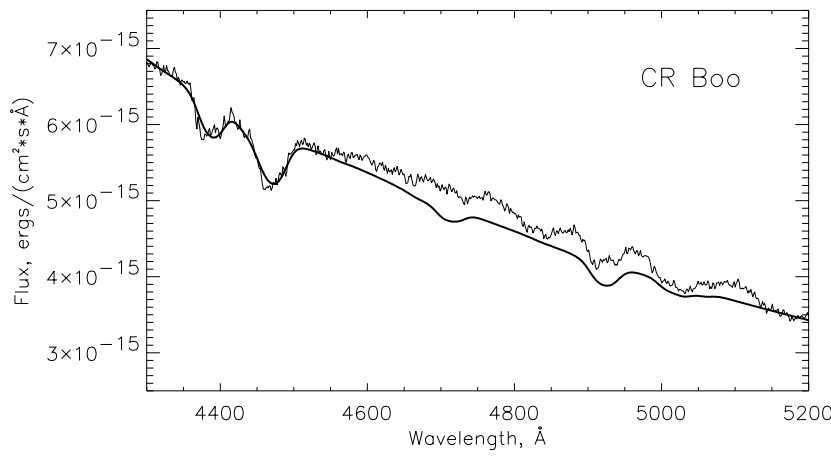

Fig. 18. The best NLTE model (solid line) which fits the observed spectrum "gc040020" (thick line) of CR Boo with parameters expected: $M_{1}=1.0 M_{\odot}, \dot{M}=4 \times 10^{-9} M_{\odot} / \mathrm{yr}, R_{\text {in }}=$ $1.4 R_{1}, R_{\text {out }}=15 R_{1}, i=30^{\circ}$.

are certainly needed to study the instability of this system in more detail.

\section{2. $C R$ Boo}

CR Boo (= PG 1346-082) is a blue star classified as an AM CVn object by Wood et al. (1987), who presented spectroscopy and high-speed photometry and demonstrated that the star is a short-period cataclysmic binary in which the mass transferred through the accretion disc is predominantly helium rather than hydrogen. A WET campaign identified a coherent period of $1471 \mathrm{~s}$, which is most likely the orbital period (Provencal et al. 1997)

Figure 18 shows the NLTE model which best fits the observed spectrum "gc040020" of CR Boo when the system shows rising brightness; other possible similar fits can be obtained within the following range of parameters: $\dot{M}=(4 \pm 1) \times 10^{-9} M_{\odot} / \mathrm{yr}, R_{\text {out }}=(15 \pm 2) R_{1}$ and $i=\left(30^{\circ} \pm 5^{\circ}\right)$ with the mass of the central star being fixed at $1.0 M_{\odot}$ and $R_{\text {in }}=1.4 R_{1}$. The fit obtained with these values indicates that the distance of CR Boo from the Earth is $470 \pm 50 \mathrm{pc}$ if no interstellar absorption is assumed. The excess of the flux in the region $\lambda 4550-5200 \AA$ may be due to flux reflected from the secondary object. The best fit is obtained with the disc radius of $\approx 15 R_{1}$ which indicates that, at the time of the observation, the disc radius was between the tidal radius and the critical radius. This means that the system was in a rising state, which we have already discovered from observation and mentioned in Sect. 6.2.

\subsection{HP Lib $(E C 15330+1403)$}

This star was identified as an AM CVn star by O'Donoghue et al. (1994). Its fundamental period, found to be $1119 \mathrm{~s}$, is close to the fundamental period of AM CVn itself, and its lack of change, in addition to a spectrum with similar broad absorption features, indicate that this is also an object stuck in its high state. A superhump period has not yet been detected (Aminzade et al. 1999).

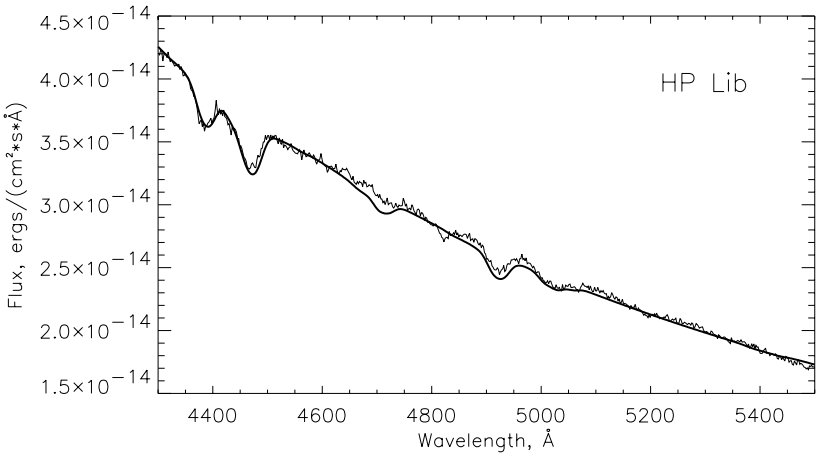

Fig. 19. The best NLTE model (solid line) which fits the observed spectrum "gc030024" (thick line) of HP Lib with parameters: $M_{1}=1.1 M_{\odot}, \dot{M}=4 \times 10^{-9} M_{\odot} / \mathrm{yr}, R_{\text {in }}=$ $1.4 R_{1}, R_{\text {out }}=14 R_{1}, i=28^{\circ}$.

Figure 19 shows the pure helium NLTE model which best fits the observed spectrum "gc030024" of HP Lib. Other combinations of parameters which give similar fits are: $\dot{M}=(4 \pm 1) \times 10^{-9} M_{\odot} / \mathrm{yr}, R_{\text {out }}=(14 \pm 3) R_{1}, i=$ $\left(28^{\circ} \pm 5^{\circ}\right)$, with the mass of the central star being fixed at $1.1 M_{\odot}$ and $R_{\text {in }}=1.4 R_{1}$. The fits obtained with these values indicate that the distance to HP Lib is $188 \pm 50 \mathrm{pc}$ if no interstellar absorption is assumed.

\section{4. $A M C V n$}

AM CVn was found to have broad, shallow absorption He I lines and no traces of hydrogen, and was first classified as a DB white dwarf (Greenstein \& Matthews 1957). Five years later, photometric variability with a period of 17.5 min was discovered; Smak (1967) and Paczyński (1967) suggested that it could be a semi-detached pair of degenerate objects in which mass transfer is driven by loss of angular momentum due to gravitational wave radiation. Flickering, which is typical for cataclysmic variables, was found by Warner \& Robinson (1972), and this inspired Faulkner, Flannery \& Warner (1972) to propose the double-degenerate white dwarf model, which has been the main accepted explanation for the AM CVn systems since then. The dominant photometric period is $525 \mathrm{~s}$, which is a first harmonic of the fundamental period of $1051 \mathrm{~s}$ (Solheim et al. 1998). A precession period of $13.38 \mathrm{hr}$ was detected by Patterson et al. (1993). Doppler tomography has established $1028.73 \mathrm{~s}$ as the binary period. (Nelemans et al. 2001b).

Figure 20 shows the NLTE pure helium model which best fits the observed spectrum "gc010043" of the AM CVn system. Other possible combinations of parameters which can also give a possible fit are: $\dot{M}=(3 \pm 1) \times$ $10^{-9} M_{\odot} / \mathrm{yr}, R_{\text {out }}=(15 \pm 2) R_{1}$ and $i=45^{\circ} \pm 5^{\circ}$, with the mass of the central star being fixed at $1.1 M_{\odot}$ and $R_{\text {in }}=1.4 R_{1}$. The fit obtained with these values indicates that the distance to the AM CVn system is $288 \pm 50 \mathrm{pc}$ if no interstellar absorption is assumed. This distance is close to the distance determined from the trigonometric parallax 


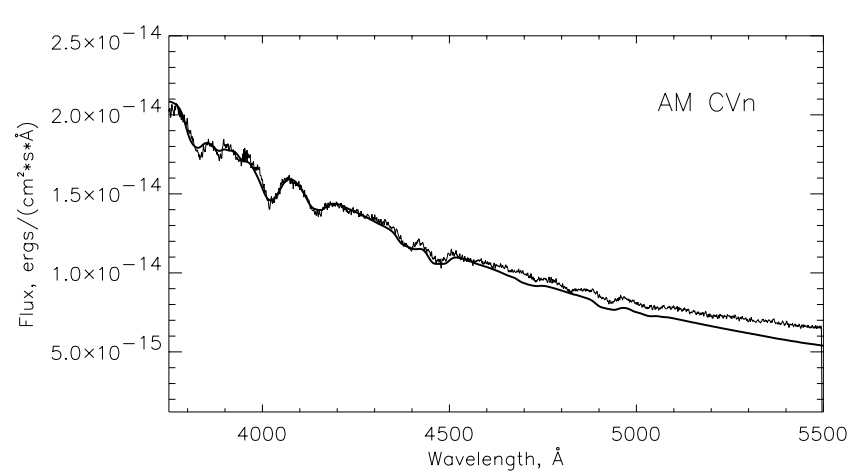

Fig. 20. The best NLTE model (solid line) which fits the observed spectrum "gc010043" (thick line) of AM CVn with parameters expected for the system to be $M_{1}=1.1 M_{\odot}, \dot{M}=$ $3 \times 10^{-9} M_{\odot} / \mathrm{yr}, R_{\text {in }}=1.4 R_{1}, R_{\text {out }}=15 R_{1}, i=45^{\circ}$ and $d=288$ pc.

program of the U.S. Naval Observatory; $235 \pm 26$ pc (Dahn, private communication).

The model fits the observed spectrum well in the region $\lambda \lambda(3700-4800) \AA$ while it diverges gradually when approaching the IR part. The same is noticed when fitting the observed spectrum of CR Boo, as shown in Fig. 18. This means that a source of energy with a temperature 7000-10 $000 \mathrm{~K}$ may be present. The secondary star, with mass $\sim 0.084 M_{\odot}$, is the only source of energy which may contribute in this region of the spectrum of AM CVn. The contribution from the secondary star has never been definitely detected, although indications of its presence have been found in the spectrum. The secondary star is believed to be sub-luminous, with effective temperature maybe only a few hundreds K (Savonije et al. 1986), but must be irradiated to a much higher temperature (Nymark \& Solheim 1995) because of its closeness to the central white dwarf and the disc.

Since our disc spectra are quite close to blackbody disc spectra, we used the blackbody-disc spectra calculations to find, as a first approximation, the temperature of the secondary star in the AM CVn system. We constructed a spectrum consisting of a primary star surrounded by a disc of $15 R_{1}$ having the same values of parameters as used to fit the observed spectrum of the AM CVn in Fig. 20. We compared this with the IUE spectrum of Solheim (1992), as shown in Fig. 21, extended to the optical and IR regions by $U, B, V, R$ and $I$ photometric points (Massacand \& Solheim 1995), which represents the most complete wavelength coverage published to date of the AM CVn system.

In Fig. 21, dotted lines show the flux of a set of rings forming the disc. Dashed-dotted lines show the spectra of the central star with a temperature of $1.0 \times 10^{5} \mathrm{~K}$ while the upper two dashed lines represent from the bottom the total disc spectrum and the spectrum of both the disc and the white dwarf, respectively. The squares are the photometric points. It is easy to see that the disc spectrum does not fit the observed spectrum in the UV and in the IR parts. This confirms the need for adding a hot (central)

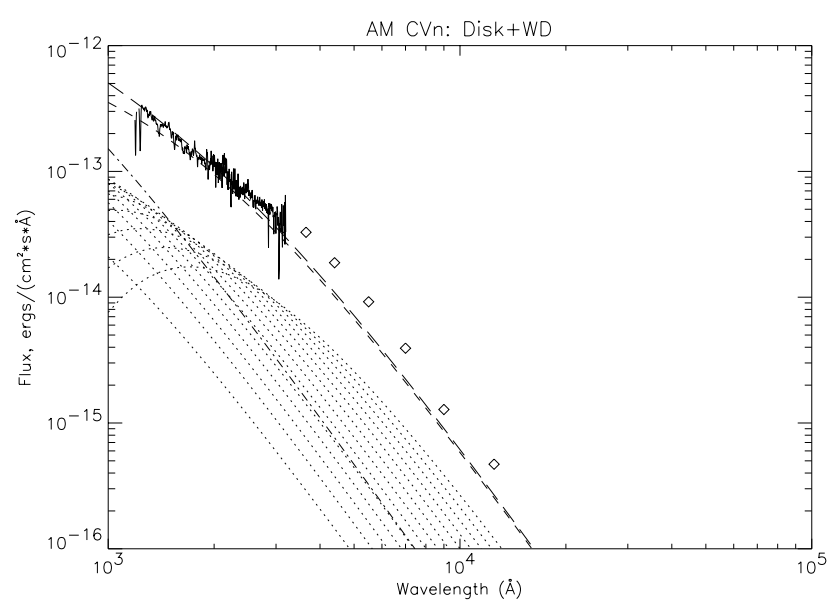

Fig. 21. The best model (upper dashed line) of the "disc + the central star" which fits the observed IUE spectrum and photometric observations (thick line + squares) of $\mathrm{AM} \mathrm{CVn}$ with parameters: $M_{1}=1.1 M_{\odot}, \dot{M}=3 \times 10^{-9} M_{\odot} / \mathrm{yr}, R_{\text {in }}=$ $1.4 R_{1}, R_{\text {out }}=15 R_{1}, i=45^{\circ}$ and the $T_{\text {eff }}=1 \times 10^{5} \mathrm{~K}$ for the central star. The other lines are explained in the text.

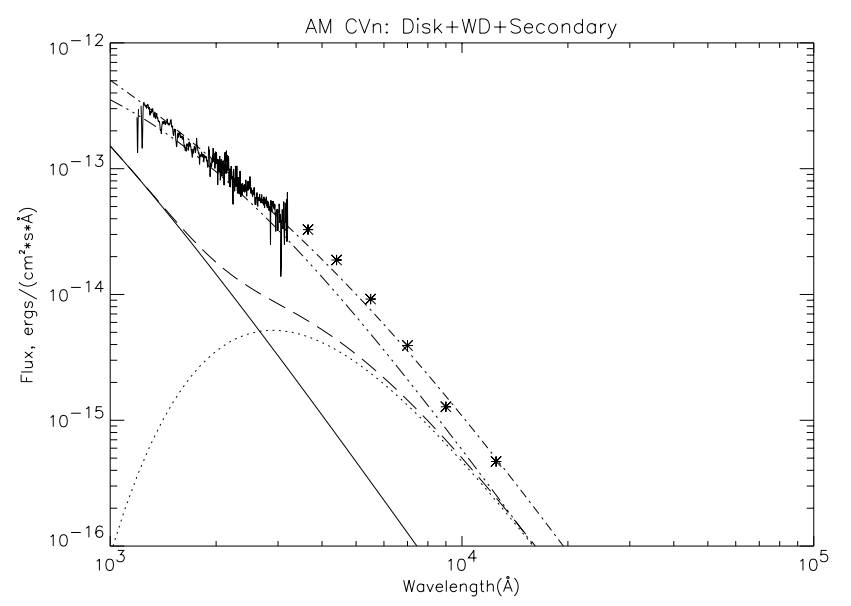

Fig. 22. The best model (upper dashed dotted line) which fits the observed IUE spectrum (thick lines + stars) of AM CVn with parameters expected for the system to be $M_{1}=$ $1.1 M_{\odot}, \dot{M}=3 \times 10^{-9} M_{\odot} / \mathrm{yr}, R_{\text {in }}=1.4 R_{1}, R_{\text {out }}=15 R_{1}$, $i=45^{\circ}, T_{\text {eff }}=10000 \mathrm{~K}$ for the secondary star while for the central star $T_{\text {eff }}=1.0 \times 10^{5} \mathrm{~K}$. The other lines are explained in the text.

star to fit the UV part, and a secondary star in the IR part.

We have added the flux of the secondary star of a Roche lobe radius, to the spectra of both the disc and the central star, with the same values of parameters stated under Fig. 21, and we found that the best fit in the IR part to the total spectrum can be found with $T_{\text {eff }}=10000 \mathrm{~K}$ for the secondary star, as seen in Fig. 22.

In this figure the dotted line represents the flux of the secondary star with $T_{\text {eff }}=10000 \mathrm{~K}$ while the dark solid line represents the flux of the central star with $T_{\text {eff }}=1.0 \times 10^{5} \mathrm{~K}$. The dashed line represents the combined flux of both the central star and the secondary star. The lower dashed-dotted line represents the flux of the 
disc while the upper dashed-dotted line represents the total combined flux of the central star, the accretion disc, and the secondary star fitted with the observed spectrum of the system and the photometric points.

We have found that our value of $T_{\text {eff }}$ for the secondary star is close to the one obtained by Nymark \& Solheim (1995), while for the central star our estimate differs. They obtained $T_{\text {eff }}=1.5 \times 10^{5} \mathrm{~K}$ for the central star and $T_{\text {eff }}=$ $12000 \mathrm{~K}$ for the secondary, because they used different values for the physical parameters of the disc, like $\dot{M}=1 \times$ $10^{-9} M_{\odot}, i=40^{\circ}$ with disc size of $\approx 15 R_{1}$ and a distance of 125 pc, while we used the values presented in Fig. 20. The present results for the parameters of the secondary star are only preliminary. If the object is substantially irradiated by the primary star and by the disc, there would be a wide distribution of $T_{\text {eff }}$ over the stellar surface. The spectrum of the secondary star may thus be quite different from the single black-body flux distribution.

\section{Discussion}

Spectra of stationary geometrically thin, optically thick pure helium accretion discs in the AM CVn systems have been investigated. Computations of synthetic spectra were done for both LTE and NLTE cases. A similar work by La Dous (1989) for solar composition discs has been used as the main reference for our work. Input parameters were chosen in such a way that we let the parameters which had largest influence on the spectrum vary, while the others were kept constant. As concluded by La Dous (1989) we found that the parameters $M_{1}$ and $\dot{M}$ have much the same effect on the spectrum, as is obvious from Eq. (8). The angle of inclination has a determining effect on the character of the observable continuous spectrum as well as the line profile. Two out of five parameters in the spectrum computation, the mass of the central star and the outer radius of the disc, cannot be determined directly from the spectroscopic observations. On the other hand, three other parameters, the mass accretion rate $\dot{M}$, the angle of inclination $i$ and the inner radius of the disc can be determined from spectroscopic observations. As also concluded by La Dous (1989), we found that the first two parameters can be determined from the optical part of the spectrum, while the last one has a very small effect in this region, and may only be determined from the UV part of the spectrum.

Based on these conclusions, we have decreased the number of free parameters in the model calculations as much as possible to be able to obtain a good fit to the observed spectra of the AM CVn systems. As mentioned in the previous sections, the mass of the central star was set in the model as a fixed parameter. The variations of the outer radius were very limited and determined based on the thermal-tidal-instability model of Tsugawa \& Osaki (1997) for the unstable objects, while for the stable objects we used the work done by Solheim et al. (1998). Finally we ended up with only two varying parameters, the mass accretion rate and the angle of inclination, which are considered as the only two parameters which can be determined from the optical spectroscopic observations and our good fits for the observed spectra confirm that. The blue shifted edges noticed in the observed spectra of V803 Cen and CR Boo at $\lambda 4370 \AA$ and $\lambda 4470 \AA$ are due to the precession of the disc, which makes the line profile vary with the orbital rotation of the system and depend on the time of observations. The same has been noticed in the set of observed spectra of the AM CVn system presented by Nelemans et al. (2001b).

In our calculations we found that both the H-He model with mass ratio $\mathrm{H} / \mathrm{He}=10^{-5}$, and the pure helium model gave a similar satisfactory fit to the observed spectra. This lead us to the conclusion that the accretion discs of the AM CVn systems most likely consist of pure helium, while the hydrogen has left the system (via stellar wind in the commom envelope phase or another mechanism). Heavy elements may also exist in the disc, but higher resolution data are certainly needed to confirm that. Similar work has been done by El-Khoury \& Wickramasinghe (2000) for some of the AM CVn systems, but only with LTE models. They applied a $\chi^{2}-$ minimization technique to estimate several system parameters for the two systems, AM CVn and CR Boo. In their work they let almost all the disc parameters be free, ignoring the limits from other observations which may be considered while setting the model grid. Leaving all the parameters free will certainly increase the level of uncertainty in the final results, especially when leaving both the mass of the central star and the outer radius of the disc free. For example, they considered the mass of the central stars as a free parameter and determined it to be $0.84 M_{\odot}$. In contrast, we have used a fixed value of mass, $\approx 1.1 M_{\odot}$, based on independent evidence, such as the mass-radius relation for a semi-degenerate secondary and the estimated mass ratio $q$ from photometric observations.

This difference in the mass can change the entire calculation quite significantly and give different values for the parameters than we obtained above. The close agreement between our calculated spectroscopic distance and the parallactic distance of AM CVn gives additional support for our procedure and the accuracy of the derived parameters of the studied systems. The values of both the mass accretion rate $\dot{M}$ and the angle of inclination $i$, obtained as a result of our calculations, are also different from that obtained by El-Khoury \& Wickramasinghe (2000), likely because we have used different masses and consequently different disc sizes. The difference in the values obtained for CR Boo can also be due to different reasons, most likely due to the instability of the system and because of the different disc radius assumed in both our and their calculations, which will certainly affect the values of both $\dot{M}$ and $i$. The assumption of LTE for their calculations and NLTE for our calculations can also give slightly different results.

The mass transfer rate we have determined is related to particular spectra and may not represent the real transfer rate for the objects. We should therefore increase the 


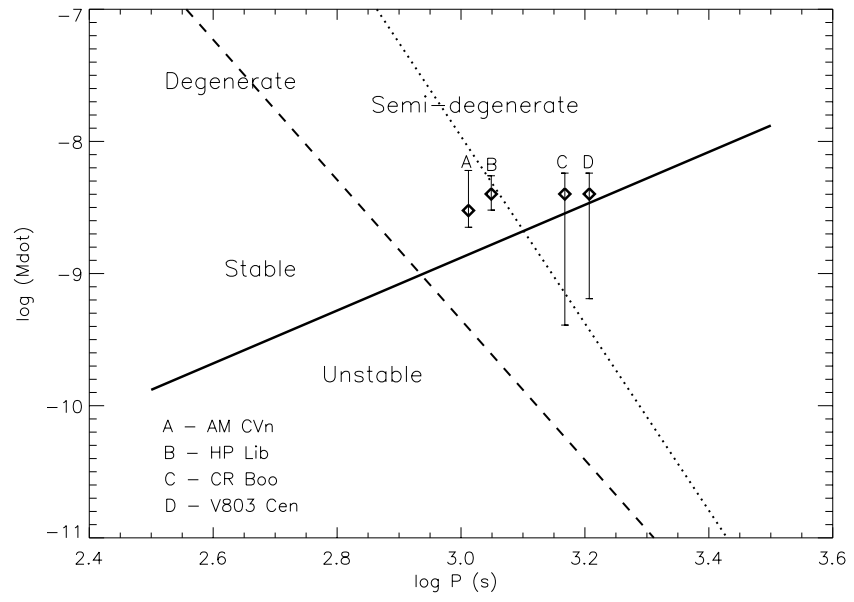

Fig. 23. The mass transfer rate versus period for the AM CVn objects observed. The error bars represent the systematic errors, due to the observations done at a particular phase. The solid line is the border between the thermally stable (upper part) and unstable discs (lower part) (Tsugawa \& Osaki 1997). The dashed line is the evolution calculated for a system with a white dwarf secondary and the dotted line for a system with a semi-degenerate pure helium secondary (Nelemans et al. 2001a).

errors when necessary. For AM CVn we have considerable variations in the line profiles during the $P=13.32 \mathrm{hr}$ period of precession, and we therefore increase the error by 50 percent. For HP Lib we have not found any sign of precession, so we keep the errors as they are. For CR Boo, the magnitude varies between 13 and 18 (Warner 1995b), we then expect $\log (\dot{M})$ to vary on the order of two units, between the high and low state for the star. The spectrum analysed for CR Boo was taken when it was about magnitude 15 , and since this system most of the time is fainter, we expect the real $\dot{M}$ to be lower than we observed. We therefore estimate an error in $\log (\dot{M})$ to be +0.15 and -1.0. For V803 Cen the magnitude range is 4.2 , which corresponds to 1.7 in $\log (\dot{M})$ variations. We observed it when fairly bright, and estimate an error in $\log \dot{M}$ to be +0.1 to -0.8 . In Table 4 we show the calculated and estimated values for $\dot{M}$ and $i$, including systematic errors. For the inclination we use the errors from the fitting by eye, except for AM CVn, where the error is increased by 50 percent due to the precession period changes. Our mass estimate, with ranges reflecting the mass variations possible from the spectral fitting taken together with the estimate, is found to be very similar to the values presented in Table 2, with possible variations in $M_{1}$ found to be $\approx 0.05 M_{\odot}$ in either system.

Since the interacting binary model was first proposed by Faulkner et al. (1972), many studies of the evolution and stability of these system have taken place. It is now commonly agreed that the orbital period will increase with time due to mass transfer, which again will decrease with time. Depending on the nature of the secondary star, i.e. if it has a $\mathrm{C}-\mathrm{N}$ core or is a pure helium object, we will get different values of the $\log \dot{M}$ versus $\log P$ relation. In
Table 4. Parameters for four of the AM CVn systems, as a result of our calculations, with no interstellar absorption assumed.

\begin{tabular}{lllll}
\hline Object & $\log (\dot{M})$ & $i$ & $R_{\text {disc }}$ & $d$ \\
Name & $M_{\odot} /$ yr & degrees & $R_{1}$ & pc \\
\hline AM CVn & $-8.5 \pm 0.3$ & $45 \pm 5$ & $(15 \pm 2)$ & $288 \pm 50$ \\
HP Lib & $-8.4 \pm 0.13$ & $28 \pm 5$ & $(14 \pm 3)$ & $188 \pm 50$ \\
CR Boo & $-8.4(+0.15-1.0)$ & $30 \pm 5$ & $(15 \pm 3)$ & $469 \pm 50$ \\
V803 Cen & $-8.4(+0.1-0.8)$ & $5 \pm 5$ & $(17 \pm 3)$ & $405 \pm 50$ \\
\hline \hline
\end{tabular}

Fig. 23 we show two such relations taken from Nelemans et al. (2001a), the dashed line showing the evolution if the secondary is a fully degenerate white dwarf, and the dotted line if the secondary is a semi-degenerate pure helium star. Our results support the semi-degenerate helium star model.

In Fig. 23 we have also drawn the border between the thermally stable and unstable mass transfer rates, as calculated by Tsugawa \& Osaki (1997). We find that AM CVn and HR Lib are stable as expected, and that the mass transfer rate of CR Boo and V803 Cen were too high to be unstable when we observed them, but on average they must belong to the unstable regime.

\section{Conclusions}

Our main conclusions are the following:

1 - The NLTE pure helium accretion disc model is a good model to compare with the observed spectra of the AM CVn systems. At the same time, small traces of hydrogen, with a hydrogen to helium mass ratio not more than $10^{-5}$, can also be introduced into the pure helium model. We found that these traces of hydrogen don't improve the final fit to the observed spectra when compared to the fit from the pure helium model. This means that the accretion disc of the AM CVn systems can be a pure helium or a helium disc with some traces of hydrogen of not more than $10^{-5}$ in mass with respect to helium. We also include the possibility that all the hydrogen was removed from the system at earlier evolutionary stages, leaving a pure helium disc.

2 - We have found that differences between the predicted LTE and NLTE equivalent widths of helium lines are between 10 and 40\%. Thus, we have demonstrated that NLTE effects are important, and should be taken into account in accurate analyses of the observed spectra of the AM CVn systems.

3 - Our results correspond with the result obtained for the H-He abundance by Iben \& Tutukov (1986) and to a certain extent with the results obtained by El-Khoury \& Wickramasinghe (2000) for the rest of the 
parameters, given that we have different assumptions and considerations.

4 - The results obtained for $\dot{M}$ calculated with a certain mass are in full agreement with the results obtained by the evolutionary models of Nelemans et al. (2001b) supporting the model of a semi-degenerate helium star as the secondary object. Parameters for four of the AM CVn systems as a result of our calculations are presented in Table 4.

Finally, further investigations will be done in the future to study the unstable systems in the low state to be able to determine the average mass transfer rates of such systems, in order to test the evolutionary models.

Acknowledgements. We thank Dr. I. Hubeny for use of his programs and kind advice during this work, and extremely valuable comments during the referee process.

\section{References}

Aminzade, R., Sullivan, D. J., Jiang, X., et al. 1999, in 11th European Workshop on White Dwarfs, ed. J.-E. Solheim, \& E. G. Meištas (San Francisco: ASP), ASP Conf. Ser., 169,305

Auer, L. H., \& Mihalas, D. 1971, ApJ, 24, 193

Crivellari, L., Hubeny, I., \& Hummer, D. G. 1991, Stellar Atmospheres: Beyond Classical Models, NATO ASI Ser. C152 (Dordrecht: Kluwer)

Elvius, A. 1975, A\&A, 44, 117

El-Khoury, W., \& Wickramasinghe, D. 2000, A\&A, 358, 154

Faulkner, J., Flannery, B. P., \& Warner, B. 1972, ApJ, 175, L79

Greenstein, J. L., \& Mathews, M. S. 1957, ApJ, 126, 14

Hubeny, I. 1988, Comp. Phys. Comm., 52, 103

Hubeny, I. 1990a, ApJ, 315, 632

Hubeny, I. 1990b, IAU Coll, 129, ed. C. Bertout et al. (Gif-surYvette: Édition Frontières), 227

Hubeny, I., Lanz, T., \& Jeffery, C. S 1994, Newsletter on Analysis of Astronomical Spectra, No. 20, ed. C. S. Jeffery (St Andrews Univ.), 30

Hubeny, I., \& Lanz, T. 1995, ApJ, 439, 875

Kudritzki, R. B., \& Hummer, D. G. 1990, ARA\&A, 28, 303
Iben, I., \& Tutukov, A. 1986, ApJ, 742, 752

La Dous, C. 1989, A\&A, 211, 131

Lynden-Bell, D., \& Pringle, J. E. 1974, MNRAS, 168, 603

Massacand, C., \& Solheim, J.-E. 1995, Baltic Astron., 4, 378

Mihalas, D. 1978, Stellar Atmospheres (San Francisco: Freeman)

Nelemans, G., Yungelson, L. R., Portegies Zwart, S. F., \& Verbunt, F. 2001a, A\&A, 365, 491

Nelemans, G., Steeghs, D., \& Groot, P. J. 2001b, MNRAS, in press

Nymark, T. K., \& Solheim, J.-E. 1995, Baltic Astron., 386, 395

O'Donoghue, D., Kilkenny, D., Chen, A., et al. 1994, MNRAS, 271,910

O'Donoghue, D., Menzies, J. W., \& Hill, P. W. 1987, MNRAS, 227,347

Paczyński, B. 1967, Acta Astron., 17, 287

Patterson, J., Halpern, J., \& Shambrok, A. 1993, ApJ, 419, 803

Provencal, J. L., Winget, D. E., Nather, E. R., et al. 1997, ApJ, 480, 383P

Robinson, E. L., Marsh, T. R., \& Smak, J. I. 1992, in Advanced Ser. in Astrophysics and Cosmology, vol. 9, ed. J. C. Wheeler, 75

Savonije, G. J., de Kool, M., \& Van Heuvel, E. P. J. 1986, A\&A, 155, 51

Semionovas, D. 1998, Master Thesis, University of Troms $\varnothing$

Semionovas, D., \& Solheim, J.-E. 1999, in 11th European Workshop on White Dwarfs, ed. J.-E. Solheim, \& E. G. Meištas (San Francisco: ASP), ASP Conf. Ser., 169, 350

Shakura, N. I., \& Sunyaev, R. 1973, A\&A, 24, 337

Smak, J. I. 1967, Acta Astron., 17, 255

Solheim, J.-E. 1992, Evolutionary processes in interacting binary stars, ed. Y. Kondo, R. F. Sisterǒ, \& R. S. Polydan (Kluwer: Dordrecht), 461

Solheim, J.-E., Provencal, J. L., Bradley, P., et al. 1998, A\&A, 332,939

Tsugawa, M., \& Osaki, Y. 1997, PASJ, 49, 75

Warner, B., \& Robinson, E. L. 1972, MNRAS, 159, 101

Warner, B. 1995a, in Cataclysmic Variables Stars (Cambridge U. Press)

Warner, B. 1995b, ApSS, 225, 249

Wood, M. A., Winget, D. E., Nather, R. E., \& Hessman, F. V. 1987, ApJ, 313, 757 